\title{
A Risk Calculator to Predict the Need for Maternal or Neonatal Hospital-Based Peripartum Intervention: Modelling National Surveillance Data
}

\author{
George Zhang ${ }^{1}$, Frances Wang ${ }^{2}$, Ha Vi Nguyen ${ }^{2}$, Jessica Bienstock ${ }^{2}$, and Marielle Gross ${ }^{3}$ \\ ${ }^{1}$ Johns Hopkins Medicine School of Medicine \\ ${ }^{2}$ Johns Hopkins University School of Medicine \\ ${ }^{3}$ University of Pittsburgh School of Medicine
}

December 22, 2020

\begin{abstract}
Objective: Given growing interest in alternatives to hospital birth, particularly given the COVID-19 pandemic, we developed a peripartum intervention risk calculator (PIRC) to estimate maternal and neonatal risk of requiring hospital-based peripartum intervention. Design: National cohort study. Setting: United States. Sample: Hospital births captured by the Pregnancy Risk Assessment Monitoring System from 2009-2018. Methods: The cohort was stratified by receipt of hospital-based interventions, defined as: 1) operative vaginal delivery (forceps or vacuum), 2) cesarean delivery, or 3) requiring neonatal intensive care unit admission. Gravidas with prior cesarean delivery or fetal malformation were excluded. Main Outcome Measures: Risk of requiring hospital-based intervention. Results: A total of 63,234 births were evaluated ( $72.6 \%$ full-term, $48.5 \%$ nulliparous) including $37.9 \%$ who received one or more hospital-based interventions. Gestational age was the most predictive factor of requiring hospital-based intervention, with lowest odds at 400/7-406/7 weeks. Previous live births (Ref: none; 1 , OR 0.41; 2 , OR 0.35; [?]3, OR 0.29; $<<0.05$ for all) were protective. Other predictors included advanced maternal age, high pre-pregnancy body mass index, maternal diabetes, maternal hypertension, and not exercising during pregnancy. The resulting seven-factor model demonstrated strong discrimination (optimism corrected C-statistic $=0.776$ ) and calibration (mean absolute error $=0.009$ ). Conclusions: We developed and validated the PIRC for predicting individualized risk for hospital-based intervention among gravidas based on seven readily accessible prenatal factors. This calculator can support personalized counseling regarding planned birth setting, helping to close a critical gap in current clinical guidance and providing an evidence-based risk assessment for those contemplating alternatives to hospital birth.
\end{abstract}

\section{Hosted file}

BJOG_Birth Calculator_Mansucript.pdf available at https://authorea.com/users/384141/articles/ 500104-a-risk-calculator-to-predict-the-need-for-maternal-or-neonatal-hospital-basedperipartum-intervention-modelling-national-surveillance-data

\section{Hosted file}

BJOG_Tables.pdf available at https://authorea.com/users/384141/articles/500104-a-riskcalculator-to-predict-the-need-for-maternal-or-neonatal-hospital-based-peripartumintervention-modelling-national-surveillance-data 
A

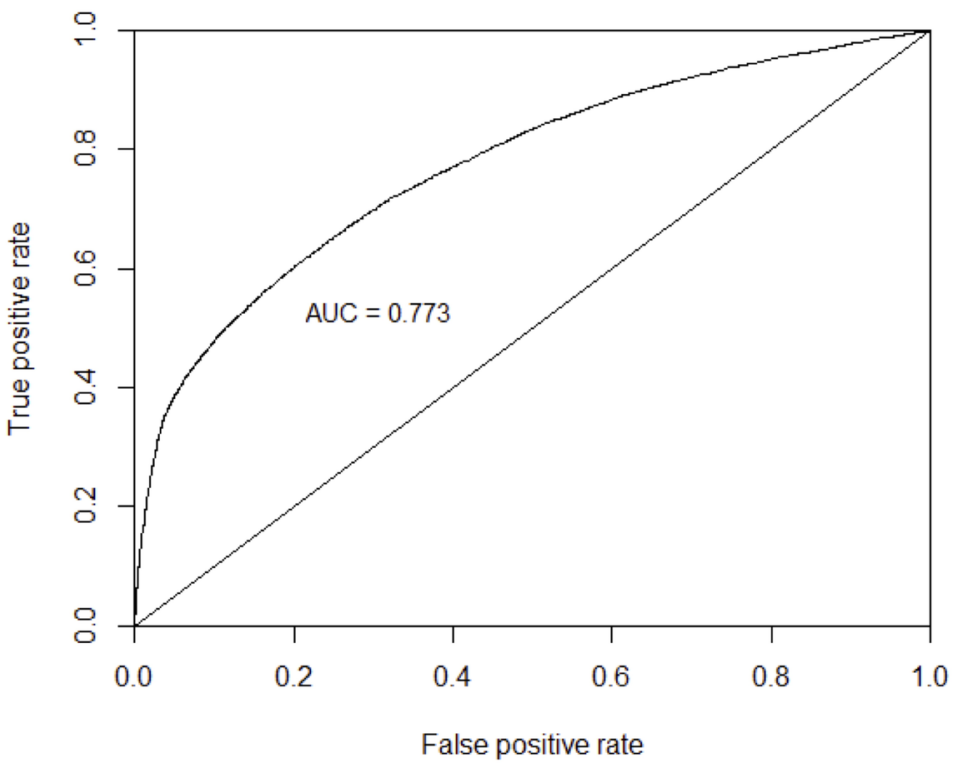

B

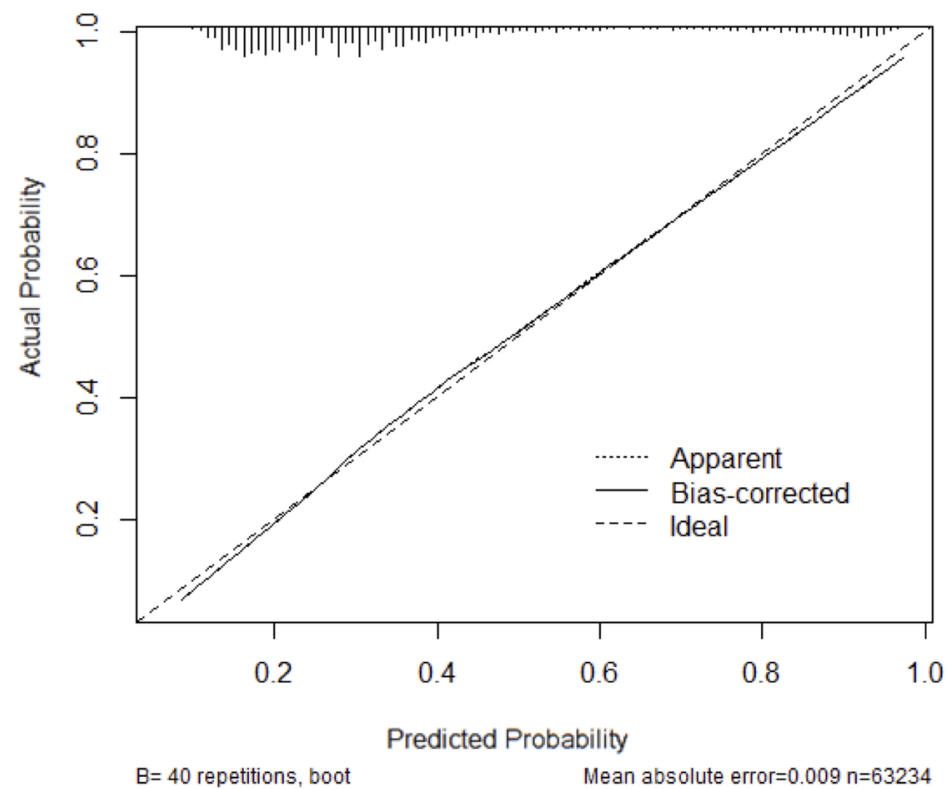




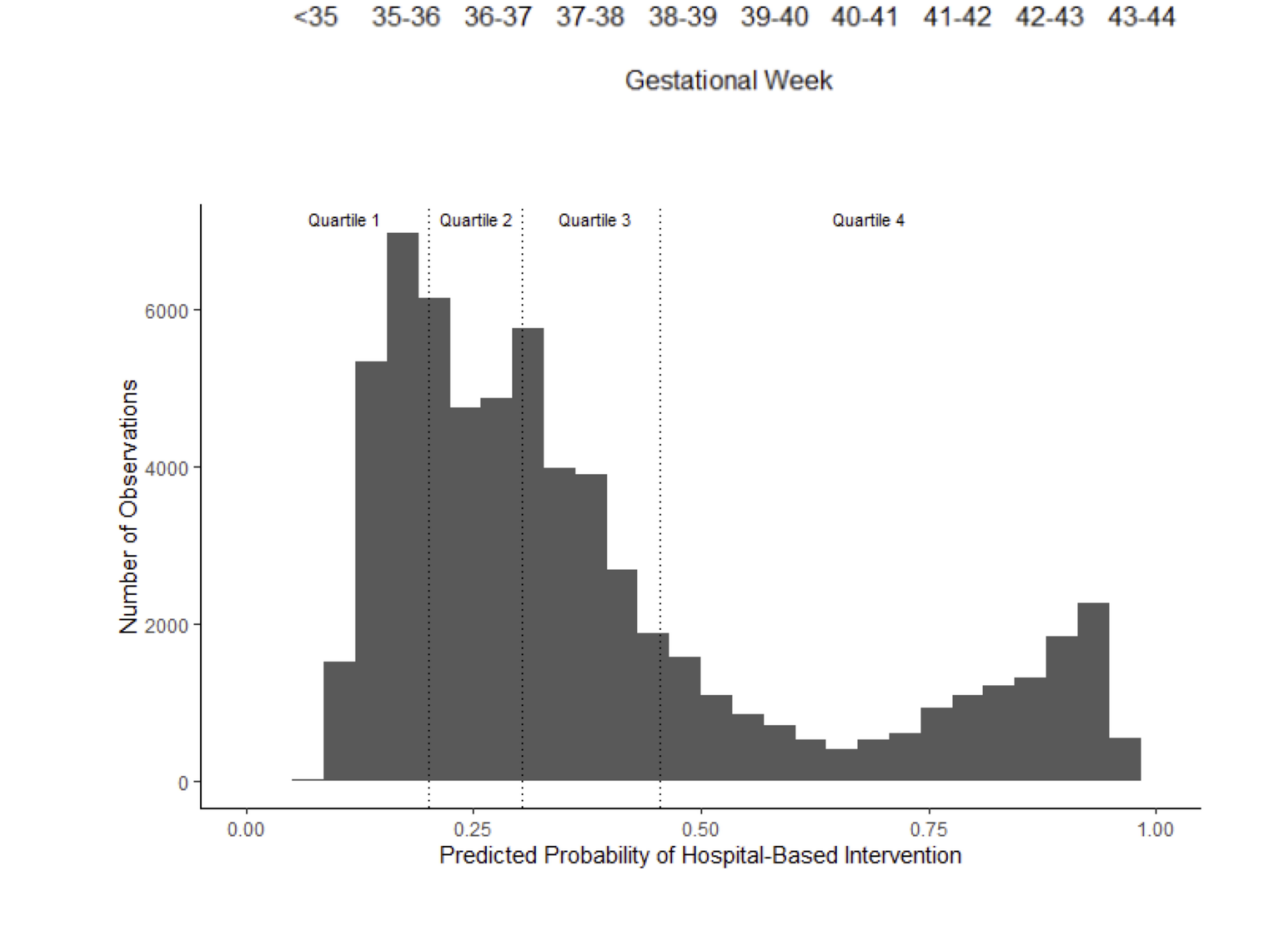




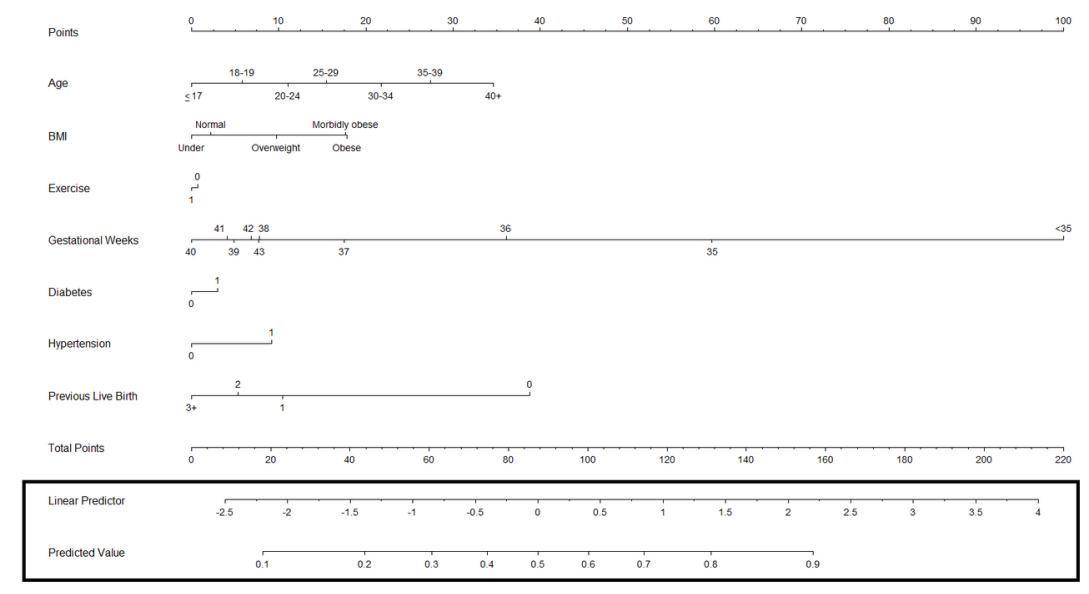

